\title{
Modified Heidelberg technique of pancreatic anastomosis postpancreaticoduodenectomy - 10 years of experience
}

\author{
Ramachandra Chowdappa,Ajeet Ramamani Tiwari, Namrata Ranganath', Rekha V. Kumar ${ }^{2}$
}

\begin{abstract}
Context: Pancreatic fistula has forever been a dreaded complication after pancreatic anastomosis (PA). We present a retrospective analysis of 10 years of experience with the Modified Heidelberg technique (MHT) that has been recently described.Aim: The aim of the study is to establish postoperative pancreatic fistula (POPF) rates after MHT. Settings and Design: This is a retrospective observational study carried out at a tertiary cancer center in South India in the Department of Surgical Oncology. Subjects and Methods: Two hundred and eight consecutive patients who underwent pancreaticoduodenectomy (PD) and PA with MHT for a variety of proximal pancreatic lesions from January 2008 to February 2018 were included in this study. The incidence of POPF was recorded by the International Study Group on Pancreatic Fistula 2005 and 2016 definitions. Statistical Analysis Used: Epidemiological and clinical data are expressed in ratios and percentage and presented in table format. Results: Between January 2008 and March 2016 , I86 patients underwent PD, and MHT was used for PA. Five (2.7\%) patients developed Grade A POPF whereas Grades B and C were seen in three (I.6\%) patients each with one death. Between April 2016 and February 2018, 22 patients underwent PD. Two patients (9\%) had biochemical leak whereas none of them developed clinically relevant POPF. No deaths were recorded in this period. Overall, Grade B and Grade C POPF rates were I.4\% each, whereas 30 -day mortality was $0.4 \%$. Conclusions: Results of this study indicate that MHT is a safe, reliable, easy to learn, and adopt technique of pancreatic reconstruction after PD.

Key words: Modified Heidelberg technique, pancreatic anastomosis, pancreatic fistula, pancreaticoduodenectomy, periampullary carcinoma
\end{abstract}

\section{Introduction}

Pancreatic anastomosis (PA) has always been a perplexing issue since Allen O. Whipple first adopted a systematic approach to pancreaticoduodenectomy (PD). ${ }^{[1]}$ The gradual improvement in safe anesthesia and intensive care fostered the state of art postoperative management in todays' contemporary high-volume institutes dealing with pancreatic cancer. This further incited surgeons to incessantly toil to improve surgical techniques most cardinal of which was a safe and secure PA.

Pancreaticoenteric anastomosis is the Achilles heel of PD. Pancreatic fistulas have always been a dreaded complication after PA with current literature showing incidence somewhere between $3 \%$ and $45 \%$ probably because multiple techniques of pancreatic reconstruction have been described, and few authors have candidly reported their anastomotic dehiscence rates. ${ }^{[2]}$ The consideration of multiple techniques of anastomosis, however, only points to the supposition that no one technique is irrevocably better than the other. Nevertheless, the detailed duct-to-mucosa anastomosis pioneered by the Heidelberg group in 2002 and later described by Shrikhande et al. in 2007 continued to be one of the most commonly used techniques against which many other techniques were compared during the past decade. ${ }^{[3,4]}$

Of late, we read an adept article by Torres et al. describing a modified Heidelberg technique (MHT) in 17 patients. ${ }^{[2]} \mathrm{We}$ were buoyant to read this article as it describes a technique that we have been using since 2008 . In the past 10 years (2008-2018), we have used this technique sans publication in 208 patients post-PD for a sundry of pancreatic head lesions. The article by Torres et al. has tempted us to present our experience with the same technique that has been a routine reconstruction strategy at our institute. The important aspect of our data representation was inclusion of the International Study Group on Pancreatic Surgery (ISGPS) 2005 definition of pancreatic fistula and its modification later in 2016. ${ }^{[5,6]}$

\begin{tabular}{|l|}
\hline Access this article online \\
\hline Quick Response Code: \\
\\
Website: www.sajc.org \\
\hline DOI: $10.4103 /$ sajc.sajc_241_18 \\
\hline
\end{tabular}

Departments of Surgical Oncology, 'Anaesthesia and Pain Relief, ${ }^{2}$ Pathology, Kidwai Cancer Institute, Bengaluru, Karnataka, India. Correspondence to: Dr.Ajeet Ramamani Tiwari, E-mail: drajeetramantiwari@gmail.com

\section{Subjects and Methods}

A total of 208 consecutive patients more than 18 years of age who underwent PD and PA with MHT for a variety of proximal pancreatic lesions from January 2008 to February 2018 were included in this study. Surgery was performed by a single surgeon, and hence, the uniformity of the technique was preserved in every case. The incidence of postoperative pancreatic fistula (POPF) was recorded by the International Study Group on Pancreatic Fistula (ISGPS) 2005 and 2016 definitions. Institution's Ethics Committee approval was taken for collection of retrospective data. Informed consent was obtained from all individuals included in the study in the preoperative period to use their clinical data for research purpose without breach of identity.

\section{Surgical technique}

We do not prefer artery first approach and rely to a great extent on good preoperative contrast-enhanced computed tomography assessment and diagnostic laparoscopy for determining operability. Due care is taken to avoid damage to pancreatic capsule during dissection as this gives a good anchorage to sutures during PA. Mesopancreatic triangle is cleared for better R0 resection. ${ }^{[7]}$ After standard pancreatic mobilization, bile duct and stomach/duodenum are divided, and the pancreatic head is prepared for transection. The jejunum is transected at the end.

Critical steps of MHT are described in Figures 1 and 2. A suitable-sized infant feeding tube is used as a pancreatic duct stent depending on the duct diameter. Two to three centimeter of the distal end of the stent outside the cut edge of pancreatic duct is cut for adequate drainage. Placement of stent at this step helps the surgeon to avoid taking the opposite wall of the duct while taking anterior and posterior ductal sutures. Its role in reducing POPF is controversial.

This is an open access journal, and articles are distributed under the terms of the Creative Commons Attribution-NonCommercial-ShareAlike 4.0 License, which allows others to remix, tweak, and build upon the work non-commercially, as long as appropriate credit is given and the new creations are licensed under the identical terms.

For reprints contact: reprints@medknow.com

How to cite this article: Chowdappa R, Tiwari AR, Ranganath $\mathrm{N}$, Kumar RV. Modified Heidelberg technique of pancreatic anastomosis postpancreaticoduodenectomy - 10 years of experience. South Asian J Cancer 2019;8:88-91. 


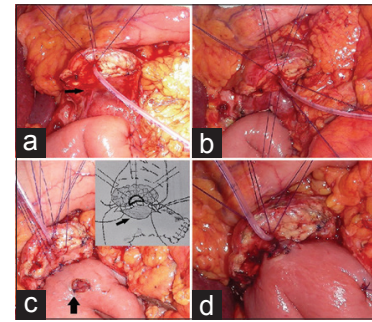

Figure 1: (a) The pancreatic remnant is mobilized for approximately $2 \mathrm{~cm}$ from the underlying splenic vein (arrow). Full-thickness three sutures at 10,12, and 2 o'clock are taken from outside (pancreatic parenchyma) to inside (pancreatic duct) without tying the knot. (b) Full-thickness sutures are taken from outside (pancreatic parenchyma) to inside (pancreatic duct) at 4, 6, and 8 o'clock position. (c) Five to six interrupted 4-0 PDS sutures are taken between posterior surface of pancreatic parenchyma and seromuscular layer of jejunum. (inset-large size of jejunal opening [horizontal arrow] in original Heidelberg technique), vertical arrow - jejunal mucosa fixation suture. (d) Needles of sutures taken on 4,6 , and 8 o'clock are passed from inside out of the jejunum to make sure the knots lie outside the anastomosis

The jejunum should lie tension-free under the cut end of the pancreas avoiding any telescopic effect at the end of anastomosis [Figure 1c]. The jejunal mucosa is fixed onto the seromuscular layer at 6 and 12 o'clock by small sutures cut just above the knot [Figure 1c (arrow)]. This avoids the retraction of jejunal mucosa and keeps its slightly everted during anastomosis. We fix the stent with pancreatic parenchyma close to the duct at its exit with 4-0 PDS suture. Two reinforcing sutures are placed at the upper and lower edge of pancreas and jejunum which help to decrease the tension on anastomosis.

Hepaticojejunostomy (HJ) is done with interrupted 4-0 PDS sutures approximately 15-20 $\mathrm{cm}$ from PA, and gastrojejunostomy/duodenojejunostomy is done in antecolic fashion in two layers about $40-45 \mathrm{~cm}$ from HJ. We do not routinely perform Braun jejunojejunostomy. The mesocolic rent is fixed over the jejunal seromuscular stitch. Two drains are placed near the anastomosis on both sides such that their tips cross beneath the anastomosis. An omental flap is placed beneath the anastomosis over superior mesenteric vessels.

POPF was defined as drainage of any measurable volume of fluid with an amylase content $>3$ times the serum amylase activity on or after postoperative day 3 , according to the criteria of the ISGPS 2005 for all patients till March 2016. ${ }^{[5]}$ However, from April 2016, the new definition was used as a drain output of any measurable volume of fluid with an amylase level $>3$ times the upper limit of institutional normal serum amylase activity, associated with a clinically relevant development/condition related directly to the POPF. Hence, the former "Grade A POPF" was redefined and called a "biochemical leak" because it had no clinical importance and was no longer referred to a true pancreatic fistula. POPF Grades B and C are confirmed but defined as more clinically relevant fistula.
In particular, Grade B requires a change in the postoperative management; drains are either left in place $>3$ weeks or repositioned through endoscopic or percutaneous procedures. Grade C POPF refers to those POPF that requires reoperation or lead to single or multiple organ failure and/or mortality due to the pancreatic fistula. ${ }^{[6]}$ Tables 1 and 2 display the results conforming to both the definitions.

\section{Results}

Consecutive 208 patients underwent PD for a variety of proximal pancreatic lesions. Males (138) were almost double compared to females (70) with average age 50.5 years (24-77), 34\% of patients presented with diabetes mellitus and $80.7 \%$ of the patients had BMI $<25 \mathrm{~kg} / \mathrm{m}^{2}$. About $78.8 \%$ patients belonged to the American Society of Anesthesiologists Score $<3$ [Table 3].

Between January 2008 and March 2016, 186 patients were operated for lesions listed in Table 1 of which periampullary carcinoma (85.5\%) was the most common. Five (2.7\%) patients developed Grade A POPF, whereas Grades B and C were seen in three $(1.6 \%)$ patients each. One patient $(0.53 \%)$ who developed Grade C POPF died due to sepsis. Three patients with Grade B fistula were managed conservatively with drain in situ and was removed after 3 weeks in two patients and 5 weeks in one patient. Two patients with Grade C fistula required prolong ventilatory support and intensive care unit admission with multiple organ failure. None of the patients required reoperation [Table 1].

Between April 2016 and February 2018, 22 patients underwent PD. Similarly, periampullary lesions $(77.27 \%)$ were the most common. Two patients $(9 \%)$ had biochemical leak, whereas none of them developed clinically relevant POPF [Table 2]. Overall, Grades B and C POPF rates were $1.4 \%$ each whereas 30 -day mortality was $0.4 \%$.

\section{Discussion}

Torres et al. in 2017 modified the original Heidelberg technique of pancreaticojejunostomy described by the Heidelberg group and later by Shrikhande et al. and published their data of 17 patients. ${ }^{[2-4]}$ We have been using a similar technique although with minor technical differences since 2008 in consecutive 208 patients.

The most important change that has been made in the modified technique described by Torres et al. is use of a much smaller jejunal lumen [Figure 1c vertical arrow] compared to original Heidelberg technique [Figure 1c (inset horizontal arrow)] and avoidance of pushing the entire raw surface of pancreatic cut end into the jejunal lumen by meticulously creating duct to mucosa anastomosis and reinforcing this with seromuscular stitches along with anchorage stitches at the end of anastomosis. ${ }^{[2]}$

The biochemical leak reported by Torres et al. is much higher $(23.5 \%)$ compared to what we recorded $(3.3 \%)$. However, in the light of its lost clinical relevance in the new ISGPS, 2016 definition, biochemical leak comparison has no clinical pertinence. What is pretty staggering is the zero clinically relevant POPF rates observed by the authors using the same technique in which we found overall $2.8 \%$ incidence of POPF. However, in the past 2 years, none of our patients 
Table 1: Grades of postoperative pancreatic fistula and mortality after modified Heidelberg technique of pancreaticojejunostomy performed from January 2008 to March 2016 as per definition of the International Study Group on Pancreatic Surgery, 2005

\begin{tabular}{lcccc} 
Final pathology & $\begin{array}{c}\text { Number } \\
\text { of cases }\end{array}$ & ISGPS (2005) leaks (January 2008-March 2016) & C \\
\cline { 3 - 5 } Periampullary & & Death & \\
$\quad$ Duodenum & 10 & 0 & 1 & 1 \\
Cholangiocarcinoma & 32 & 2 & 0 & 0 \\
Pancreatic & 21 & 2 & 1 & 0 \\
Ampullary & 96 & 0 & 0 & 0 \\
Pancreatic head & 11 & 1 & 0 & 1 \\
Neuroendocrine & 9 & 0 & 0 & 0 \\
Chronic pancreatitis & 3 & 0 & 0 & 0 \\
Cystic neoplasm & 4 & $5(2.7)$ & $3(1.6)$ & 0 \\
Total (\%) & 186 & & 0 & 0 \\
\hline
\end{tabular}

ISGPS=International study group on pancreatic surgery

Table 2: Grades of postoperative pancreatic fistula and mortality after modified Heidelberg technique of pancreaticojejunostomy performed from April 2016 to February 2018 as per definition of the International Study Group on Pancreatic Surgery, 2016

\begin{tabular}{lcccc}
\hline Final pathology & $\begin{array}{c}\text { Number } \\
\text { of cases }\end{array}$ & \multicolumn{2}{c}{\begin{tabular}{c} 
ISGPS (2016) leaks (April 2016-February 2018) \\
\cline { 3 - 4 }
\end{tabular}} & $\begin{array}{c}\text { Biochemical leak } \\
\text { (clinically not relevant) }\end{array}$ \\
\hline Periampullary & & 1 & 0 & Grade B \\
$\quad$ Duodenum & 1 & 0 & 0 & 0 \\
Cholangiocarcinoma & 1 & 0 & 0 & 0 \\
Pancreatic & 13 & 1 & 0 & 0 \\
Ampullary & 3 & 0 & 0 & 0 \\
Pancreatic head & 1 & 0 & 0 & 0 \\
Neuroendocrine & 0 & 0 & 0 & 0 \\
Chronic pancreatitis & 1 & 0 & 0 & 0 \\
Cystic neoplasm & 22 & $2(9)$ & 0 & 0 \\
Total (\%) & & 0 & 0 \\
\hline
\end{tabular}

ISGPS=International study group on pancreatic surgery

Table 3: Demographic data of patients undergoing pancreaticoduodenectomy from January 2008 to February 2018

\begin{tabular}{lcc}
\hline Demographic parameters & Subdivision & Total \\
\hline Age (years) & - & $50.5(24-77)$ \\
Sex ratio (male:female) & - & $2: 1(138: 70)$ \\
Diabetes (\%) & - & $71(34)$ \\
BMI $\left(\mathrm{kg} / \mathrm{m}^{2}\right)(\%)$ & $<25$ & $168(80.7)$ \\
& $>25$ & $40(19.3)$ \\
ASA (\%) & I & $43(20.6)$ \\
& II & $121(58.2)$ \\
& III & $42(20.2)$ \\
& IV & $2(1)$ \\
\hline
\end{tabular}

$\mathrm{ASA}=$ American society of anesthesiologists score, $\mathrm{BMI}=$ Body mass index

have developed clinically relevant POPF [Table 2]. Although there were few minor technical changes between ours' and Torres et al.'s technique such as all the knots in the inner layer remained outside the anastomosis, anterior and posterior outer layers were interrupted sutures instead of continuous, decrease in the length of stent and fixing the stent to pancreatic parenchyma, no robust comparison can be drawn considering the small number of patients (17) analyzed by Torres et al. ${ }^{[2]}$

Büchler et al. in January 2000 published their results of Heidelberg technique in 331 patients with POPF incidence of $2.1 \%{ }^{[8]}$ However, they were not classified as per the ISGPS definition as it came into being only 5 years later in 2005 . Our clinically relevant POPF incidence was $2.8 \%$. Its very 90 difficult to compare with studies and techniques between those developed and published in pre-ISGPS definition of POPF era and those that were developed later. Only a well-conducted randomized trial comparing MHT with the original Heidelberg technique would help us draw any major conclusions.

Our technique has one of the lowest rates of POPF rates when compared to other contemporary techniques developed after 2005 like the binding PJ (Grade B $-7.2 \%$ and Grade C $-10.2 \%$ ) and invagination $\mathrm{PJ}$ (clinically relevant POPF $-10 \%$ ). ${ }^{[9,10]}$ Although very encouraging results have been published by Yang et al. with their Colonial Wig PJ technique with no clinically relevant fistula, only 22 patients were included in this comparative study, and a much larger study would be required to find what really transpired such low POPF rates. ${ }^{[1]}$

The drawback of this study is that there is no comparative group of another anastomotic technique and that it is a retrospective study. However, one foremost conclusion that can be drawn from our study is reduction of clinically relevant POPF rates from 2.8\% (2008-2016) to 0\% (2016-2018) in past 2 years [Tables 1 and 2]. This does suggest that it is just not the technique but also surgeon's experience and attention to meticulous details during resection and anastomosis that has tremendous bearing on postoperative course of these patients.

\section{Conclusions}

Results of this study indicate that MHT is a safe, reliable, easy to learn, and adopt the technique of pancreatic reconstruction after South Asian Journal of Cancer • Volume 8 •Issue 2 • April-June 2019 
PD. However, it is important to understand that experience with one particular technique and obsessive compulsion to scrupulously adhere to finer details of the surgery is more consequential.

\section{Financial support and sponsorship}

Nil.

\section{Conflicts of interest}

There are no conflicts of interest.

\section{References}

1. Griffin JF, Poruk KE, Wolfgang CL. Pancreatic cancer surgery: Past, present, and future. Chin J Cancer Res 2015;27:332-48.

2. Torres OJ, Costa RC, Costa FF, Neiva RF, Suleiman TS, Souza YL, et al. Modified Heidelberg technique for pancreatic anastomosis. Arq Bras Cir Dig 2017;30:260-3.

3. Z'graggen K, Uhl W, Friess H, Büchler MW. How to do a safe pancreatic anastomosis. J Hepatobiliary Pancreat Surg 2002;9:733-7.

4. Shrikhande SV, Kleeff J, Büchler MW, Friess H. Pancreatic anastomosis after pancreaticoduodenectomy: How we do it. Indian J Surg 2007;69:224-9.

5. Bassi C, Dervenis C, Butturini G, Fingerhut A, Yeo C, Izbicki J, et al.
Postoperative pancreatic fistula: An international study group (ISGPF) definition. Surgery 2005; 138:8-13.

6. Bassi C, Marchegiani G, Dervenis C, Sarr M, Abu Hilal M, Adham M, et al. The 2016 update of the International Study Group (ISGPS) definition and grading of postoperative pancreatic fistula: 11 years after. Surgery 2017; 161:584-91.

7. Chowdappa R, Challa VR. Mesopancreas in pancreatic cancer: Where do we stand-review of literature. Indian J Surg Oncol 2015;6:69-74.

8. Büchler MW, Friess H, Wagner M, Kulli C, Wagener V, Z'Graggen K, et al. Pancreatic fistula after pancreatic head resection. Br J Surg 2000;87:883-9.

9. Casadei R, Ricci C, Silvestri S, Campra D, Ercolani G, D’Ambra M, et al. Peng's binding pancreaticojejunostomy after pancreaticoduodenectomy. An Italian, prospective, dual-institution study. Pancreatology 2013; 13:305-9.

10. Senda Y, Shimizu Y, Natsume S, Ito S, Komori K, Abe T, et al. Randomized clinical trial of duct-to-mucosa versus invagination pancreaticojejunostomy after pancreatoduodenectomy. Br J Surg 2018; 105:48-57.

11. Yang X, Aghajafari P, Goussous N, Patel ST, Cunningham SC. The "Colonial wig" pancreaticojejunostomy: Zero leaks with a novel technique for reconstruction after pancreaticoduodenectomy. Hepatobiliary Pancreat Dis Int 2017; 16:545-51. 